\title{
La fluticasona nasal fue más efectiva que la loratadina oral en la rinitis alérgica estacional
}

A comparison of the efficacy of fluticasone propionate aqueous nasal spray and loratadine, alone and in combination, for the treatment of seasonal allergic rhinitis. Ratner P,Van Bavel J, Martin B. The Journal of Family Practice 1998; 47:118-125.

\section{Objetivo}

Evaluar la eficacia de fluticasona propionato spray acuoso nasal (FPSN) vs. loratadina sola vs. tratamiento combinado para el manejo de la rinitis alérgica estacional.

\section{Diseño}

Ensayo clínico multicéntrico randomizado, doble ciego, controlado por placebo. Duración: 14 días.

\section{Lugar}

Cinco centros de Estados Unidos.

\section{Pacientes}

Se incluyeron 600 pacientes de ambos sexos entre 13 y 80 años, la mayoría de raza blanca, que cumplían cuatro criterios diagnósticos para rinitis alérgica estacional: 1) Reacción cutánea positiva a antígeno de juniperus, 2) Mucosa nasal hipertrofiada, 3) Por lo menos dos episodios previos de rinitis alérgica en estaciones de polinización pasadas, 4) Tener registrado por lo menos un episodio de rinitis moderada a severa en la historia clínica médica.

\section{Intervención}

Se randomizó a los pacientes en cuatro grupos iguales ( $n=150)$ a recibir 1) FPSN $50 \mathrm{mg}$ en cada fosa nasal dos veces por día más placebo oral. 2) Placebo spray nasal más loratadina $10 \mathrm{mg}$ vía oral a las 8 de la mañana. 3) Placebo oral. 4) FPSN más loratadina $10 \mathrm{mg}$ por día.

\section{Medición de resultados principales}

El resultado principal se definió como atenuación de los síntomas de rinitis (bloqueo nasal, estornudos, conjuntivitis, etc.) y descenso en el puntaje de alteración de la calidad de vida en el curso del tratamiento y al finalizar el mismo; evaluado por autocuestionario y por examen médico.

\section{Resultados principales}

\begin{tabular}{lll}
\hline & $\begin{array}{l}\text { Mejoría significativa } \\
(\% \text { pacientes }\end{array}$ & $\begin{array}{l}\text { no cambios } \\
\text { en los síntomas }\end{array}$ \\
\hline FPSN $(n=150)$ & $42 \%$ & $4 \%$ \\
\hline FPSN +Loratadina $(n=150)$ & $46 \%$ & $2 \%$ \\
\hline Loratadina $(n=150)$ & $17 \%$ & $7 \%$ \\
\hline Placebo $(n=150)$ & $15 \%$ & $20 \%$ \\
\hline
\end{tabular}

Estos resultados evidencian que no existen diferencias significativas al comparar el tratamiento combinado de FPSN +loratadina con FPSN como única terapéutica, y que tampoco existió una diferencia significativa entre placebo y loratadina. Por otra parte F.P ans muestra buenos resultados terapéuticos comparada con placebo y con loratadina $(p<0.0 .5)$

\section{Conclusiones}

El tratamiento con FPSN en pacientes con rinitis estacional redujo significativamente los síntomas y mejoró el puntaje de calidad de vida tras 7 y 14 días de tratamiento. La adición de loratadina no aportó más beneficio.

\section{COMENTARIO}

La rinitis alérgica se presenta en dos formas: la forma crónica y la aguda estacional. En el tratamiento episódico se pueden utilizar descongestivos locales de acción corta (la seudoefedrina, la nafazolina, la fenilefrina); locales de acción prolongada (la xilometazolina y oximetazolina); descongestivos sistémicos (como la seudoefedrina, efedrina, fenilpropanolamina), antihistamínicos de segunda generación como astemizol, Loratadina y terfenadina, o corticoides.

Los descongestivos locales provocan congestión nasal de rebote y no deben usarse en exceso (en general no más de tres días). El astemizol y la terfenadina están relacionados con episodios cardiovasculares de importancia, incluyendo prolongación del intervalo QTc, torsades des pointes y otras arritmias cuando se combinan con macrólidos y otras drogas 1 . La forma crónica es debida a diferentes alergenos ambientales (polvo doméstico, caspa animal, hongos ambientales, perfumes, insecticidas, etc.) y se beneficia con un tratamiento crónico que no presente considerables efectos adversos, como los corticoides tópicos nasales. Los efectos adversos más comunes son leves e incluyen la sequedad de mucosa nasal, disfonía, candidiasis localizada y la epistaxis esporádica. Estos medicamentos pueden ser utilizados a largo plazo sin grandes riesgos de absorción sistémica considerable. Solo el uso muy prolongado y en altas dosis, podría estar relacionado con desaceleración de la velocidad de crecimiento pondo-estatural en infantes ${ }^{2}$. Otro efecto descripto para corticoides inhalados a altas dosis es la producción de glaucoma de ángulo abierto ${ }^{3-4}$. En cuanto a la rinitis aguda estacional, lo que reviste particular importancia es la prevención de los episodios. Los corticoides nasales podrían ser utilizados empezando 20 días antes del inicio de la estación de polinización, si bien esto no ha sido debidamente demostrado, abriendo un interesante campo de estudio. Otros estudios clínicos combinaron beclometasona con antihistamínicos (astemizoly terfenadina) revelando mejores resultados que la monoterapia. Se realizaron también estudios combinando beclometasona y mometasona con loratadina por 7 días y uno con fluticasona versus cetirizina oral por ocho semanas. Este último fue criticado porque los resultados de la rinitis alérgica deberían medirse a corto plazo, ya que los estudios a largo plazo están limitados por el período que dura la polinización $n^{5}$.

Este es el primer estudio que usa placebo nasal (que tendría cierto efecto de lavado de la mucosa nasal) y que evalúa fluticasona en combinación con la loratadina. Otro estudio 6 mostró que la fluticasona era superior a la loratadina, presentando los resultados en forma objetivable con flujometría nasal. En resumen, en el tratamiento de la rinitis alérgica estacional, los mejores resultados parecen obtenerse con los corticoides nasales y el tratamiento con antihistamínicos orales no parece ser mejor que el placebo.

\section{Dr. Marcelo Ikonikoff}

Unidad de Medicina Familiar y Preventiva. Hospital Italiano de Buenos Aires.

\section{Referencias}

1. F Estelle, R Simons, the Canadian Beclomethasone Dipropionate-Salmeterol Xinafoate Study Group. A Comparison of Beclomethasone, Salmeterol, and Placebo in Children with Asthma. N Engl J Med 1997;337:1659-65

2. Garbe E, LeLorier J, Boivin JF, Suissa S. Inhaled and Nasal Glucocorticoids and the Risks of Ocular Hypertension or Open-angle Glaucoma. JAMA. 1997;277:722-727

3. Babe KS, Serafin WE: Histamine, bradykinin, and their antagonist5, en Goodman \& Gilman The Pharmaceutical Basis of Therapeutics. Mc Graw Hill, $9^{\circ}$ edición 1996. pp 586-592

4. Hanania NA, Chapman KR, Kesten S.Adverse effects of inhaled corticosteroids.Am J Med 1995:98:196-208.

5. International Rhinitis Management Working Group. International consensus report on the diagnosis an management of rhinitis. Allergy 1994:49(supl.19):1-34.

6. Jordana G, Dolovich J,Briscoe MP, et al.Intranasal fluticasone propionate versus loratadine in the treatment of adolescent patients with seasonal allergic rhinitis.J Allergy Clin Inmunol 1996 97:588-95. 